\title{
Evidence for a sauropod-like metacarpal configuration in ankylosaurian dinosaurs
}

Phil Senter

Acta Palaeontologica Polonica 56 (1), 2011: 221-224 doi: http://dx.doi.org/10.4202/app.2010.0041

Ankylosaurian dinosaurs are armored, quadrupedal members of the ornithischian clade Thyreophora. Ankylosaurs are typically portrayed with the metacarpals slanted and distally divergent, with their proximal ends arranged in a shallow arc, both in the literature (Matthew 1922; Gaston et al. 2001; McCrea et al. 2001; Vickaryous et al. 2004) and in museum mounts (Fig. 1). In contrast, Carpenter (1984) illustrated the metacarpals of the ankylosaur Sauropelta edwardsorum Ostrom, 1970, from the Lower Cretaceous Cloverly Formation of Wyoming and Montana, with their proximal ends arranged in a tight, semicircular arc, but even in that depiction themetacarpals were slanted and distally divergent. Members of the thyreophoran clade Stegosauria, the sister taxon to the Ankylosauria (Butler et al. 2008), have also typically been portrayed with slanted and distally divergent metacarpals (Marsh 1891; Gilmore 1914; Galton and Upchurch 2004). Some researchers expressed the opinion that stegosaur metacarpals were held vertically, not distally divergent, with their proximal ends arranged in a tight, semicircular arc, so that the metacarpus formed a vertical half-tube (von Huene 1931; Thulborn 1990; Christiansen 1997) such that flexion of digit I would move it toward digit V. Manual manipulation of stegosaurian metacarpals has since confirmed that this is the correct configuration of the stegosaurian metacarpus (Senter 2010). Here I investigate the possibility that the ankylosaurian metacarpus exhibited a similar configuration. As in the previous study on stegosaurs (Senter 2010), I treat the slanting and spreading configuration and the vertical semi-tubular configuration as competing hypotheses, each with a set of testable predictions. Each hypothesis of metacarpal configuration in ankylosaurs predicts that the configuration (1) is allowed by the shapes of the metacarpals, (2) provides a better fit (alignment and contact of opposing articular surfaces) between the metacarpals than the competing hypothesis, (3) does not compromise the goodness of fit between the metacarpals and the phalanges, (4) is not contradicted by articulated specimens, and (5) agrees with ichnological evidence. In the previous study on stegosaurs I included an additional prediction: that the configuration provides sufficient support for and does not disarticulate the more proximal forelimb bones. Here, that prediction is omitted, because the ankylosaurian carpus is unknown (Vickaryous et al. 2004) except for a single carpal described by Maleev (1954).

Phil Senter [psenter@uncfsu.edu], Department of Biological Sciences, Fayetteville State University, 1200 Murchison Road, Fayetteville, North Carolina 28301, USA. 
This is an open-access article distributed under the terms of the Creative Commons

Attribution License (for details please see creativecommons.org), which permits unrestricted use, distribution, and reproduction in any medium, provided the original author and source are credited.

Forill text $(246.9 \mathrm{kB})$ 